\title{
Enfermedad cardiaca inducida por radioterapia
}

\section{Camilo A. Velásquez ${ }^{a, *}$, Miguel González ${ }^{\mathrm{b}}$, María Gabriela García-Orjuela ${ }^{\mathrm{c}}$ y Nicolás Jaramillo ${ }^{c}$}

\author{
a Universidad CES-Cardiovital Investigaciones, New Haven, CT, Estados Unidos \\ b Universidad CES - Cardiovital Investigaciones, Newark, NJ, Estados Unidos \\ c Cardiovital Investigaciones, Medellín, Colombia
}

Recibido el 17 de abril de 2017; aceptado el 10 de junio de 2017

Disponible en Internet el 10 de octubre de 2017

\section{PALABRAS CLAVE \\ Radioterapia; \\ Corazón; \\ Complicaciones; \\ Neoplasias; \\ Tratamiento}

\section{KEYWORDS \\ Radiotherapy; \\ Heart; \\ Complications; \\ Neoplasia; \\ Treatment}

\begin{abstract}
Resumen La enfermedad cardiaca inducida por los tratamientos para el cáncer, como la quimioterapia y radioterapia, se considera cada vez más una enfermedad prevalente, que por tanto amerita conocimiento para el seguimiento adecuado, la prevención y el tratamiento de la misma. Anteriormente, este grupo de investigación publicó el artículo "Cardiotoxicidad inducida por la quimioterapia: desde las bases moleculares hasta la perspectiva clínica", en la que se hace una revisión acerca de los diferentes aspectos, incluyendo diagnóstico, fisiopatología, tratamiento y pronóstico de la enfermedad cardiaca inducida por radioterapia.

(c) 2017 Sociedad Colombiana de Cardiología y Cirugía Cardiovascular. Publicado por Elsevier España, S.L.U. Este es un artículo Open Access bajo la licencia CC BY-NC-ND (http:// creativecommons.org/licenses/by-nc-nd/4.0/).
\end{abstract}

\begin{abstract}
Radiation induced heart disease
Abstract The heart disease induced by cancer treatments, such as chemotherapy and radiotherapy, is increasingly being seen as a prevalent disease, and as such it needs to be taken into account for its appropriate follow-up, prevention and treatment. Previously, this research group published the review, "Chemotherapy-induced cardiotoxicity from the molecular basis to the clinical perspective", which included a review of the different aspects, including the pathophysiology, treatment, and prognosis of heart disease induced by radiotherapy.

(c) 2017 Sociedad Colombiana de Cardiología y Cirugía Cardiovascular. Published by Elsevier España, S.L.U. This is an open access article under the CC BY-NC-ND license (http:// creativecommons.org/licenses/by-nc-nd/4.0/).
\end{abstract}

\footnotetext{
* Autor para correspondencia.

Correo electrónico: c_velasquezm@outlook.com (C.A. Velásquez).
} 


\section{Introducción}

Dentro del tratamiento de los pacientes con cáncer, existen dos grandes pilares terapéuticos con gran impacto en la sobrevida de los pacientes, estos son la quimioterapia (QT) y radioterapia (RT) ${ }^{1}$. Sin embargo, algunos de estos tratamientos se han asociado con aumento del riesgo de desarrollar toxicidad cardiaca, la cual puede tener manifestaciones como falla cardiaca, disfunción asintomática del ventrículo izquierdo, hipertensión arterial, arritmias, prolongación del intervalo QT, enfermedad pericárdica, enfermedad cardiaca valvular y enfermedad cardiaca isquémica ${ }^{2-4}$, que a su vez pueden ser agudas, subagudas o crónicas ${ }^{3}$.

El término cardiotoxicidad ha sido utilizado de manera general para describir la toxicidad directa o indirecta de distintos agentes, dentro de los que se encuentran diferentes xenobióticos o agentes quimioterapéuticos, así como la radiación ${ }^{1}$. En una revisión publicada previamente por este grupo de investigación, se trató de manera profunda el efecto de los agentes quimioterapéuticos sobre la función cardiaca, su clasificación y el uso racional mediante la prevención y el tratamiento ${ }^{1}$.

En el año 2002, el comité de evaluación y revisión cardiaca (Cardiac Review and Evaluation Committee) definió ciertas condiciones aclaratorias de cardiotoxicidad, para lo cual debe cumplirse con una o más de las siguientes:

- Miocardiopatía con disminución en la función ventricular izquierda que sea global o más grave hacia el septum.

- Síntomas de falla cardiaca.

- Signos asociados con falla cardiaca, incluyendo presencia de S3, taquicardia o ambos.

- Disminución de al menos el $5 \%$ de la fracción de eyección (FE) con valores menores al 55\% con signos o síntomas de la falla cardiaca, o una disminución de al menos el $10 \%$ en la FE con valores menores del $55 \%$ sin la presencia de signos o síntomas ${ }^{1,5}$.

\section{Epidemiología}

La enfermedad cardiaca inducida por radiación (ECIR) es una complicación que afecta aproximadamente a un $10-30 \%$ de los pacientes, y hasta el $88 \%$ presentan alteraciones anatómicas asintomáticas que pueden evolucionar a complicaciones clínicas serias ${ }^{6,7}$. Sin embargo, es difícil conocer con exactitud la epidemiología de la ECIR debido a los constantes cambios en las técnicas y mejoras en la dosimetría de la radiación, así como en la protección del paciente, lo cual tiende a disminuir la exposición cardiovascular. Igualmente, los efectos cardiacos suelen no ser inmediatos, sino que requieren de varios años para presentarse ${ }^{7}$.

\section{Factores de riesgo}

En la ECIR se ha considerado a la radioterapia mediastinal como el principal factor de riesgo relacionado con dicha condición. Este tipo de radioterapia es ampliamente utilizada en pacientes con diagnóstico de malignidades torácicas, como el linfoma de Hodgkin y el cáncer de mama ${ }^{7-9}$.

Otros factores de riesgo considerados para el desarrollo de ECIR incluyen: dosis acumulativas altas de radiación (>
$30 \mathrm{~Gy}$ ), edad menor a 50 años al momento de la radiación, dosis altas de fracción de radiación (> $2 \mathrm{~Gy} /$ día), protección inadecuada o ausente, historia previa de enfermedad cardiaca, presencia y extensión del tumor cerca del corazón, tratamiento concomitante con quimioterapia cardiotóxica y presencia de otros factores de riesgo cardiovasculares ${ }^{3,10-12}$.

\section{Radioterapia}

Esta incluye el uso de partículas de alta energía, rayos $x$, o rayos $\gamma$ que fragmentan el ADN celular e interfieren con la proliferación y viabilidad celular. Esto afecta principalmente las células tumorales, dado su alto índice metabólico y proliferativo. Sin embargo, se considera que dosis mayores a 30 Gy pueden generar daños en el tejido cardiaco ${ }^{13,14}$.

En los últimos años se ha hecho énfasis en exponer al paciente a la mínima radiación posible sin comprometer su pronóstico. Las tres estrategias utilizadas para disminuir la toxicidad cardiaca son la reducción de las dosis de radiación, la disminución del campo y del volumen de radiación, y la utilización de nuevas técnicas de planeación y de administración ${ }^{10}$. Fuera de las estrategias anteriormente planteadas, el uso de técnicas nuevas como la radioterapia de intensidad modulada y la terapia con haz de protones ha ayudado a disminuir la $\mathrm{ECIR}^{10}$.

\section{Fisiopatología}

Los mecanismos por los cuales la radiación genera cardiotoxicidad son diversos y sinérgicos, y generalmente toman años para manifestarse clínicamente ${ }^{14,15}$. Dentro de estos se encuentran (tabla 1):

- Inflamación aguda: mediada principalmente por el daño endotelial. Minutos después de la radiación, las células endoteliales se vuelven muy permeables. Luego de la segunda hora, el endotelio expresa moléculas unidas a la membrana como la selectina E y P, ICAM-1 y PECAM-1, implicadas en el reclutamiento y migración leucocitario, principalmente de neutrófilos, liberando citoquinas proinflamatorias como el factor de necrosis tumoral (FNT) y la interleuquina 8 (IL-8), reclutando células inflamatorias adicionales $^{7,16,17}$. Así mismo, hay depósitos tempranos de fibrina en los capilares, que alteran el proceso de fibrinólisis endógena, y generan estado de hipercoagulabilidad local ${ }^{7}$.

- Fibrosis: es el principal mecanismo del compromiso crónico. La fibrosis es el resultado del depósito anormal del colágeno por los miofibroblastos activados. La fibrosis cardiovascular es un proceso crónico y dinámico que depende de citoquinas profibróticas (IL-13, IL-4, FNT- $\beta$ ) y de la presencia de hipoxia crónica y estrés oxidativo ${ }^{7}$.

- Estrés oxidativo: se considera que desempeña un papel importante en la transición de la inflamación aguda a la fibrosis. Las especies reactivas de oxígeno son generadas de manera aguda por la acción directa de la radiación así como por los macrófagos y el endotelio inflamado. El estrés oxidativo propaga la inflamación por varios mecanismos entre los que se encuentran la quimiotaxis, el aumento de la actividad de la trombina y la interacción 
Tabla 1 Mecanismos fisiopatológicos de la enfermedad cardiaca inducida por radioterapia

\begin{tabular}{lll}
\hline Tipo de mecanismo & Fisiopatología & $\begin{array}{l}\text { Órgano } \\
\text { implicado }\end{array}$ \\
\hline Inflamación aguda & - Aumento de la permeabilidad de célula endotelial & Endotelio \\
& - Expresión de moléculas de membrana (Selectina E/P, ICAM-1, PECAM-1) & \\
& - Liberación de citoquinas proinflamatorias (FNT/IL8) & Endotelio \\
Fibrosis & - Alteración del proceso de fibrinólisis endógena & Miocardio \\
Estrés oxidativo & - Depósito anormal del colágeno por los miofibroblastos activados & Endotelio \\
& - Liberación de citoquinas profibróticas (IL-13, IL-4, FNT- $\beta$ ) & \\
& - Transición de la inflamación aguda a la fibrosis & \\
& - Propagación de la inflamación por distintos mecanismos (quimiotaxis, & \\
& aumento de la actividad de la trombina, e interacción con factor de & \\
\hline
\end{tabular}

Tabla 2 Frecuencia de las principales complicaciones cardiacas secundarias a radiación mediastinal

\begin{tabular}{ll}
\hline Tipo de complicación & $\begin{array}{l}\text { Incidencia luego de radiación } \\
\text { mediastinal }\end{array}$ \\
\hline $\begin{array}{l}\text { Compromiso pericárdico } \\
\text { Cardiopatía inducida por }\end{array}$ & $\begin{array}{l}\text { Actualmente }<5 \% \\
\text { Aproximadamente } 25 \%\end{array}$ \\
radioterapia & \\
Enfermedad valvular & $6-40 \%$ \\
Enfermedad coronaria & $1,9-7,7 \%$ \\
\hline
\end{tabular}

con el factor de necrosis $\mathrm{FN}-\kappa \beta$, responsable de la regulación inmune ${ }^{7}$.

\section{Tipo de complicaciones cardiacas postradioterapia}

La ECIR incluye distintas formas de compromiso cardíaco. Históricamente, la complicación cardiaca más común era el compromiso pericárdico, no obstante, con el uso de nuevas técnicas como la radioterapia fraccionada, esta afección ha disminuido de manera significativa. En la actualidad la complicación más común es el daño valvular, seguido del daño de otras estructuras anatómicas como el músculo cardíaco, los sistemas de conducción y las arterias coronarias (tabla 2$)^{11}$.

1. Pericarditis: Es la forma más temprana de ECIR luego de la radiación mediastinal. Auténticamente las complicaciones pericárdicas eran comunes, con una incidencia del 4 al 20\%, pero con el uso de nuevas técnicas esta se ha disminuido a menos del $5 \%{ }^{18}$. Puede ocurrir de dos formas: temprana y aguda o retardada y crónica, comportándose como dos entidades distintas desde el punto de vista histopatológico ${ }^{6,7}$.

- Forma temprana y aguda: es el resultado de altas dosis de radioterapia, considerado > 36 a 40 Gy o > 50 Gy administrado al 30\% del corazón; sin embargo, es rara en la actualidad debido a las técnicas reductoras de dosis. Puede ocurrir durante la RT o días a semanas después. Dentro de las características histopatológicas, se observa que el pericardio se torna poroso, favoreciendo la infiltración de neutrófilos y la colección de exudado rico en proteínas $^{7,18}$.
- Forma crónica: es la complicación cardiaca más común relacionada con la RT. Es dosis dependiente, con una incidencia de $>50 \%$, si la dosis supera los 50-60 Gy. El rango de tiempo de presentación está entre los tres meses siguientes a la radiación, hasta una década después. No obstante, la incidencia de esta condición ha disminuido del $20 \%$ al 2,5\% con el uso de nuevas técnicas. Desde el punto de vista histopatológico se caracteriza por engrosamiento fibroso, adherencias y reemplazo de la grasa pericárdica por colágeno. En el $20 \%$ de los casos puede presentarse como pericarditis constrictiva ${ }^{6,7,18}$.

2. Miocardiopatía inducida por radioterapia (CMIR): generalmente ocurre a una tasa de incidencia acumulada a 40 años del 24,8\%. El riesgo de desarrollar CMIR aumenta después de 5 años, pero puede progresar décadas después de la terapia, requiriéndose generalmente dosis acumuladas de $40 \mathrm{~Gy}^{7}$. En cuanto a histopatología se caracteriza por inflamación que progresa a fibrosis intersticial del miocardio en parches ${ }^{7}$. A medida que el corazón se vuelve fibrótico, pierde su distensibilidad, lo cual conlleva disfunción diastólica, seguida de trastornos de la motilidad de pared; esto ocurre entre el $18 \%$ al $29 \%$ de los pacientes en la segunda a tercera décadas luego del tratamiento con $\mathrm{RT}^{7,17}$.

3. Enfermedad valvular: esta complicación depende de la dosis de la radiación, y por extensión, de la década en la cual fue tratado el paciente, debido a los protocolos utilizados entre 1965 y $1995^{7}$. Se considera que cerca del 6 al 40\% de los pacientes que reciben RT en el mediastino, presentan complicaciones valvulares ${ }^{19}$, pero más del $70 \%$ de las personas afectadas permanecen asintomáticas ${ }^{10}$. Estas son dependientes de la dosis de radiación y aumentan exponencialmente con dosis superiores a $30 \mathrm{~Gy}$. En lo que respecta a la histopatología, se caracteriza porque durante los primeros 10 años se forman retracciones valvulares, que generan insuficiencia valvular, principalmente en las válvulas mitral y aórtica. Posteriormente, hay progreso hacia engrosamiento fibrótico y calcificación de las válvulas, dando lugar a estenosis hasta 20 años después de la RT, siendo más frecuente en la válvula aórtica $^{7,10,19}$.

4. Enfermedad coronaria inducida por radioterapia: los pacientes con historia de RT tienen mayor riesgo de 
eventos coronarios hasta 20 años después de la exposición. Este riesgo varía entre 1,9 a $7,7 \%$ más que la población general ${ }^{20}$. Histopatológicamente se caracteriza por lesiones más extensas, que comprometen principalmente el ostium. La arteria descendente anterior es la arteria coronaria más afectada debido a su proximidad con el campo de radiación. La placa, a diferencia de la producida por el proceso de aterosclerosis, es más fibrosa, con disminución en el componente lipídico $^{7,10}$. Es considerada la principal causa de muerte en pacientes llevados a $\mathrm{RT}^{10}$.

5. Arritmias y enfermedad del sistema de conducción: la presencia de arritmias fatales, así como enfermedades del sistema de conducción, ocurre años luego del tratamiento. Existe un amplio espectro de alteraciones incluidos el síndrome de nodo sinusal enfermo y todas las formas de bloqueos aurículo-ventriculares y de rama, los cuales se reconocen con facilidad en los electrocardiogramas de rutina ${ }^{6,10,21}$. El bloqueo de rama derecha es la alteración de conducción más frecuente debido a la proximidad con el endocardio. La RT torácica también se ha asociado con prolongación del intervalo $\mathrm{QT}^{10}$.

\section{Monitorización de los pacientes llevados a radioterapia}

Antes del inicio de la radioterapia se recomienda una evaluación completa del paciente para definir su riesgo cardiovascular basal ${ }^{13}$.

En los pacientes asintomáticos de alto riesgo, definidos como aquellos que recibieron dosis mayores a $30 \mathrm{~Gy}$, pacientes con factores de riesgo cardiovascular, o aquellos que recibieron QT concomitante, se recomienda realizar ecocardiografía cada 5 años ${ }^{22}$. Así mismo, en este grupo, debido al alto riesgo de eventos coronarios, se recomienda hacer pruebas de inducción de isquemia no invasiva 5 a 10 años después de la $\mathrm{RT}^{22}$.

El seguimiento de los pacientes asintomáticos de bajo riesgo, se hace mediante ecocardiografía transtorácica 10 años después de la evaluación inicial ${ }^{22}$ (fig. 1).

La ecocardiografía desempeña un rol principal en la evaluación cardiaca estructural y funcional de los pacientes llevados a radioterapia. Sin embargo, existen otras técnicas más avanzadas, que incluyen tomografía computarizada cardiaca, resonancia magnética cardiaca y medicina nuclear ${ }^{17}$.

Durante el 2013, el consenso de expertos de imagen multimodal para la evaluación de las complicaciones cardiacas de la radioterapia, publicó las recomendaciones para la realización de cada uno de los exámenes mencionados ${ }^{17}$ :

- Ecocardiografía: permite evaluar alteraciones cardiacas estructurales y la severidad de la enfermedad valvular, así como medir la función del ventrículo izquierdo. De acuerdo con las indicaciones clínicas, pueden utilizarse distintas técnicas como el modo $M$, Doppler, 2D/3D transtorácico o transesofágico, con contraste, o la ecocardiografía de estrés ${ }^{17,23}$.

- Ventajas: alta disponibilidad y reproducibilidad

- Desventajas: variabilidad intra- e interobservador, la calidad de la imagen está condicionada por la tecnología del

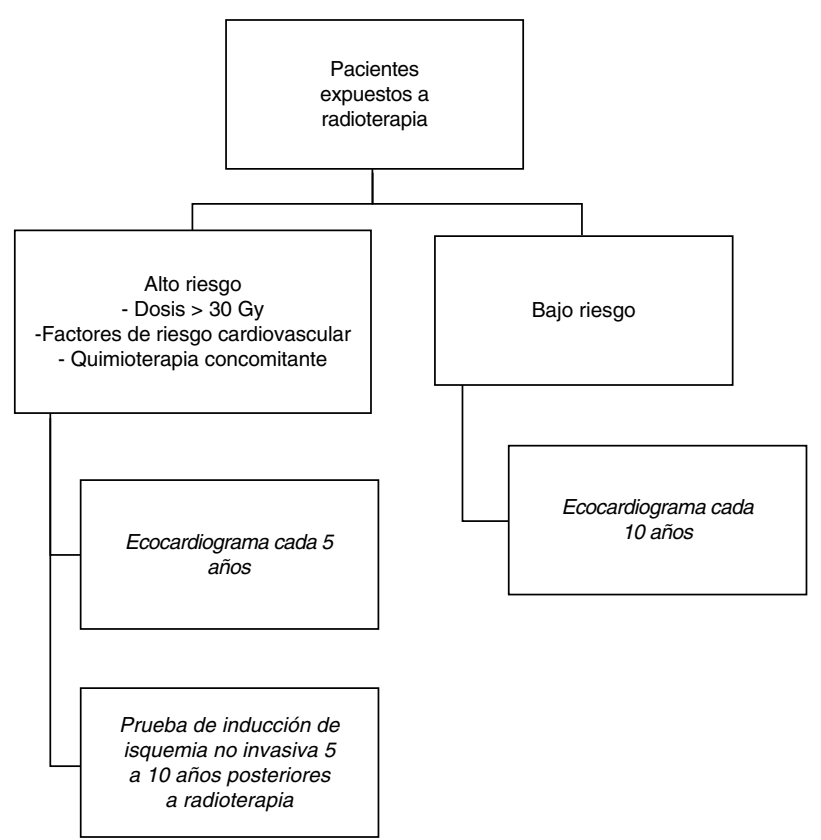

Figura 1 Algoritmo de seguimiento para pacientes con exposición a radioterapia.

equipo. Valor predictivo dudoso para la detección temprana del daño subclínico ${ }^{3}$.

La ecocardiografía con strain longitudinal, como método de detección de trastornos de la contractilidad, ha surgido como una nueva herramienta más sensible para identificar disfunción ventricular izquierda sutil y subclínica, debido a que el subendocardio es más susceptible que las capas subepicárdicas, a una variedad de enfermedades ${ }^{24}$.

- Resonancia magnética cardiaca: provee una visión excelente de la morfología del corazón, pericardio, grandes vasos y estructuras adyacentes ${ }^{17}$.

- Ventajas: se considera el estudio estándar de oro para la evaluación de la fracción de eyección del ventrículo izquierdo. Permite la caracterización tisular en pacientes con mala ventana pericárdica. Tiene alta resolución temporal y espacial y alta reproducibilidad ${ }^{3}$.

- Desventajas: altos costos y disponibilidad limitada; claustrofobia en el paciente y dispositivos que prohiben realizar el estudio como por ejemplo los marcapasos. Riesgo de fibrosis nefrogénica sistémica y reacción alérgica secundaria al medio de contraste ${ }^{3}$.

- Tomografía computarizada cardiaca: ofrece una imagen anatómica transversal del tórax. Además, permite la evaluación con medio de contraste para la diferenciación de los tejidos, y la adquisición o la reconstrucción de imágenes según el electrocardiograma ${ }^{17}$.

- Ventajas: evaluación de estenosis coronaria y medición de calcio.

- Desventajas: exposición a radiación, resolución temporal baja. Riesgo de reacción alérgica secundaria al medio de contraste ${ }^{3}$.

- Medicina nuclear: incluye la tomografía computarizada de emisión de fotón simple y la tomografía de emisión de positrones, que utilizan diferentes tecnologías para 
proveer información que ayuda a determinar la presencia y extensión de la enfermedad cardiaca ${ }^{17}$.

- Ventajas: evaluación funcional y de perfusión miocárdica, identificación de cicatrices post-infarto de miocardio y de miocardio viable. Buena determinación de fracción de eyección de ventrículo izquierdo. Baja variabilidad intrae interobservador. Alta reproducibilidad y disponibilidad ${ }^{3}$.

- Desventajas: exposición a radiación.

\section{Prevención}

En la actualidad, en la radioterapia torácica se está dirigiendo la atención principalmente hacia la prevención de las complicaciones cardiacas relacionadas con la radioterapia, incluyéndose diferentes técnicas y recomendaciones, entre ellas ${ }^{25-27}$ :

- Fraccionamiento de radioterapia de 1,8-2 Gy por día.

- Minimización de campos de radiación en tórax.

- Maniobras de RT en inspiración profunda.

- Eliminación o disminución de factores de riesgo dependientes del paciente (tabaquismo, dislipidemia).

- Ajuste de dosis de medicamentos cuando se requiere QT/RT concomitante.

- Seguimiento clínico e imagenológico estrecho según condiciones basales, síntomas y riesgo cardiovascular del paciente.

\section{Tratamiento}

El manejo de los pacientes con enfermedad cardiaca inducida por radioterapia se basa en las recomendaciones generales de cada tipo de manifestación ${ }^{13}$.

- Enfermedad pericárdica: según el tipo de compromiso pericárdico que presente el paciente $e^{8,10}$.

o Forma aguda: incluye manejo farmacológico con antiinflamatorios no esteroideos y requerimiento de drenaje guiado por ecocardiografía (pericardiocentesis) en pacientes con derrames pericárdicos que comprometan el estado hemodinámico del paciente.

- Forma crónica: en caso de presentarse con taponamiento cardiaco, se realiza pericardiocentesis como tratamiento inicial. Si es recurrente, puede optarse por pericardiectomía.

- Miocardiopatía inducida por radioterapia: el tratamiento incluye las recomendaciones de manejo de las guías de falla cardíaca de otras etiologías, con énfasis en el manejo sintomático, por lo que como primera línea de manejo se recomiendan los inhibidores de enzima convertidora de angiotensina y los betabloqueadores ${ }^{10,13}$. Según el estadio y compromiso del paciente, pueden adicionarse medicamentos como bloqueadores de los receptores de angiotensina y antagonistas de aldosterona ${ }^{8}$. El trasplante cardiaco puede ser una opción para pacientes seleccionados con enfermedad cardiaca terminal secundaria a radiación $^{10}$.

- Enfermedad valvular: las recomendaciones de tratamiento son las mismas que en los pacientes con enfermedad valvular por otras causas $^{8}$, pero con tasas de complicaciones más elevadas que las de la población general, reportándose tasas de mortalidad asociadas a cirugía de cambio de una sola válvula del $45 \%$, aumentándose hasta $61 \%$ cuando se incluyen dos o más válvulas, comparado con el $13 \%$ y $17 \%$ en pacientes sin historia de exposición a radiación torácica, respectivamente ${ }^{10}$.

- Enfermedad coronaria inducida por radioterapia: debido a que no hay guías de manejo específicas para esta condición, se recomienda el mismo manejo de los pacientes con enfermedad coronaria de origen arteriosclerótico, incluyendo tanto el manejo farmacológico como la revascularización coronaria, quirúrgica o percutánea, dependiendo de la sintomatología del paciente, evaluación del lecho coronario, estadio del cáncer, expectativa de vida y comorbilidades ${ }^{8}$.

En general, en los pacientes con historia de exposición a radioterapia, se recomienda la revascularización percutánea sobre la revascularización quirúrgica, debido a que esta última conlleva ciertas dificultades por la fibrosis mediastinal concomitante, así como la estenosis secundaria a radiación de la arteria mamaria interna, que impiden su uso. Igualmente, muchos pacientes pueden presentar daño pulmonar concomitante inducido por radioterapia, lo que aumenta el riesgo de complicaciones pulmonares perioperatorias $^{8,10}$.

\section{Conclusiones}

Pese a que la RT ha ayudado a mejorar la sobrevida de los pacientes oncológicos, sus efectos secundarios, dentro de los que se resalta la enfermedad cardiaca, obligan al clínico a realizar una evaluación completa y un adecuado seguimiento del paciente, para prevenirlas y tratarlas a tiempo, así como a aplicar distintas técnicas y recomendaciones que ayuden a disminuir el riesgo.

Con este artículo de revisión se quiere llamar la atención a los médicos expuestos a esta población de pacientes de gran crecimiento, para que tengan en cuenta la morbilidad asociada con la exposición a la radiación y sus efectos a corto y largo plazo cuando no se logra hacer una prevención activa durante la fase crítica y un tratamiento adecuado de sus complicaciones en la fase activa de la ECIR.

\section{Responsabilidades éticas}

Protección de personas y animales. Los autores declaran que para esta investigación no se han realizado experimentos en seres humanos ni en animales.

Confidencialidad de los datos. Los autores declaran que en este artículo no aparecen datos de pacientes.

Derecho a la privacidad y consentimiento informado. Los autores declaran que en este artículo no aparecen datos de pacientes.

\section{Conflicto de intereses}

María Gabriela García trabaja con Novartis de Colombia, pero no recibió ningún tipo de participación por parte de 


\section{dicha compañía. El resto de los autores declaran no tener} ningún conflicto de intereses.

\section{Bibliografía}

1. Velasquez C, Gonzalez M, Berrouet MC, Jaramillo N. Cardiotoxicidad inducida por la quimioterapia: desde las bases moleculares hasta la perspectiva clínica. Rev Colomb Cardiol. 2016;23:104-11.

2. Marwick TH. Cancer therapy-related cardiac dysfunction: unresolved issues. Can J Cardiol. 2016;32:842-6.

3. Rosa GM, Gigli L, Tagliasacchi MI, Di lorio C, Carbone F, Nencioni A, et al. Update on cardiotoxicity of anti-cancer treatments. Eur J Clin Invest. 2016;46:264-84.

4. Aleman BMP, Moser EC, Nuver J, Suter TM, Maraldo MV, Specht L, et al. Cardiovascular disease after cancer therapy. Eur J Cancer Suppl. 2014;12:18-28.

5. Speyer J. Cardiac dysfunction in the trastuzumab clinical experience. J Clin Oncol. 2002;20:1156-7.

6. Carver JR, Shapiro CL, Ng A, Jacobs L, Schwartz C, Virgo KS, et al. American society of clinical oncology clinical evidence review on the ongoing care of adult cancer survivors: Cardiac and pulmonary late effects. J Clin Oncol. 2007;25:3991-4008.

7. Cuomo JR, Sharma GK, Conger PD, Weintraub NL. Novel concepts in radiation-induced cardiovascular disease. World J Cardiol. 2016;8:504-19.

8. Yusuf SW, Sami S, Daher IN. Radiation-induced heart disease: a clinical update. Cardiol Res Pract. 2011:1-9 (April 2007).

9. Jacob S, Pathak A, Franck D, Latorzeff I, Jimenez G, Fondard 0 , et al. Early detection and prediction of cardiotoxicity after radiation therapy for breast cancer: the BACCARAT prospective cohort study. Radiat Oncol. 2016:1-10.

10. Donnellan E, Phelan D, McCarthy CP, Collier P, Desai M, Griffin B. Radiation-induced heart disease: A practical guide to diagnosis and management. Cleve Clin J Med. 2016;83:914-22.

11. Aleman BMP, van den Belt-Dusebout AW, De Bruin ML, van 't Veer MB, Baaijens MHA, de Boer JP, et al. Late cardiotoxicity after treatment for Hodgkin lymphoma. Blood. 2007;109:1878-86.

12. Yeh ETH, Tong AT, Lenihan DJ, Yusuf SW, Swafford J, Champion C, et al. Cardiovascular complications of cancer therapy: diagnosis, pathogenesis, and management. Circulation. 2004;109:3122-31.

13. Herrmann J, Lerman A, Sandhu NP, Villarraga HR, Mulvagh SL, Kohli M. Evaluation and management of patients with heart disease and cancer: cardio-oncology. Mayo Clin Proc. 2014;89:1287-306.

14. Hancock SL, Tucker MA, Hoppe RT. Factors affecting late mortality from heart disease after treatment of Hodgkin's disease. JAMA. 1993;270:1949-55.

15. Chargari C, Riet F, Mazevet M, Morel É, Lepechoux C, Deutsch É. Complications of thoracic radiotherapy. Press Medical. 2013;42(9 PART2):e342-51.
16. Cuzick J, Stewart H, Rutqvist L, Houghton J, Edwards R, Redmond $\mathrm{C}$, et al. Cause-specific mortality in long-term survivors of breast cancer who participated in trials of radiotherapy. J Clin Oncol Off J Am Soc Clin Oncol. 1994;12:447-53.

17. Lancellotti P, Nkomo VT, Badano LP, Bergler-Klein J, Bogaert J, Davin L, et al. Expert consensus for multi-modality imaging evaluation of cardiovascular complications of radiotherapy in adults: a report from the European Association of Cardiovascular Imaging and the American Society of Echocardiography. Eur Heart J Cardiovasc Imaging. 2013;14:721-40.

18. Carmel RJ, Kaplan HS. Mantle irradiation in Hodgkin's disease. An analysis of technique, tumor eradication, and complications. Cancer. 1976;37:2813-25.

19. Wethal T, Lund M-B, Edvardsen T, Fosså SD, Pripp AH, Holte $\mathrm{H}$, et al. Valvular dysfunction and left ventricular changes in Hodgkin's lymphoma survivors. A longitudinal study. Br J Cancer. 2009;101:575-81.

20. Darby SC, Ewertz M, McGale P, Bennet AM, Blom-Goldman U, Brønnum D, et al. Risk of ischemic heart disease in women after radiotherapy for breast cancer. N Engl J Med. 2013;368: 987-98.

21. Larsen RL, Jakacki RI, Vetter VL, Meadows AT, Silber JH, Barber G. Electrocardiographic changes and arrhythmias after cancer therapy in children and young adults. Am J Cardiol. 1992;70:73-7.

22. Caro Codon J, Rosillo Rodriguez SO, Lopez Fernandez T. Cardiotoxicity from the cardiologist's perspective. Futur Cardiol. 2015;11:425-32.

23. Plana JC, Galderisi M, Barac A, Ewer MS, Ky B, Scherrer-Crosbie $M$, et al. Expert consensus for multimodality imaging evaluation of adult patients during and after cancer therapy: A report from the American society of echocardiography and the European association of cardiovascular imaging. J Am Soc Echocardiogr. 2014;27:911-39.

24. Mele D, Malagutti P, Indelli M, Ferrari L, Casadei F, Da Ros L, et al. Reversibility of left ventricle longitudinal strain alterations induced by adjuvant therapy in early breast cancer patients. Ultrasound Med Biol. 2016;42:125-32.

25. Aznar MC, Maraldo MV, Schut DA, Lundemann M, Brodin NP, Vogelius IR, et al. Minimizing late effects for patients with mediastinal Hodgkin lymphoma: deep inspiration breathhold, IMRT, or both? Int J Radiat Oncol Biol Phys. 2015;92: 169-74.

26. Specht L, Yahalom J, Illidge T, Berthelsen AK, Constine LS, Eich $\mathrm{HT}$, et al. Modern radiation therapy for Hodgkin lymphoma: field and dose guidelines from the international lymphoma radiation oncology group (ILROG). Int J Radiat Oncol Biol Phys. 2014;89:854-62.

27. Damkjær SMS, Aznar MC, Pedersen AN, Vogelius IR, Bangsgaard JP, Josipovic M. Reduced lung dose and improved inspiration level reproducibility in visually guided DIBH compared to audio coached EIG radiotherapy for breast cancer patients. Acta Oncol. 2013;52:1458-63. 